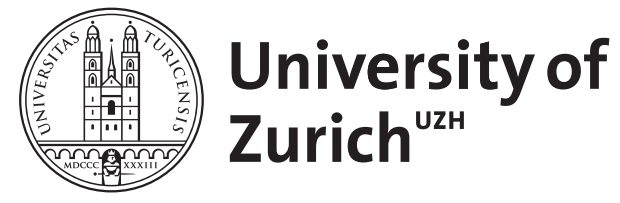
Archive

University of Zurich

University Library

Strickhofstrasse 39

CH-8057 Zurich

www.zora.uzh.ch

Year: 2006

\title{
Zur Möglichkeit einer kulturübergreifenden Bioethik
}

Schulz-Baldes, Annette ; Jakovljevic, Anna-Karina

DOI: https://doi.org/10.1007/s00481-006-0445-7

Posted at the Zurich Open Repository and Archive, University of Zurich

ZORA URL: https://doi.org/10.5167/uzh-155790

Journal Article

Published Version

Originally published at:

Schulz-Baldes, Annette; Jakovljevic, Anna-Karina (2006). Zur Möglichkeit einer kulturübergreifenden Bioethik. Ethik in der Medizin, 18(3):261-266.

DOI: https://doi.org/10.1007/s00481-006-0445-7 
Annette Schulz-Baldes · Zürich

Anna-Karina Jakovljevic · Göttingen

\title{
Zur Möglichkeit einer kulturübergreifenden Bioethik
}

\author{
Interdisziplinärer Workshop des Lehrstuhls \\ für Biomedizinische Ethik und der Arbeits- \\ und Forschungsstelle für Ethik (Universität Zürich) \\ in Kooperation mit der Jungen Akademie der Berlin- \\ Brandenburgischen Akademie der Wissenschaften und \\ Akademie der Naturforscher Leopoldina Ethik-Zentrum \\ der Universität Zürich, 30. März bis 1. April 2006
}

\section{K}

ulturelle Diversität bedeutet immer auch moralische Diversität. Moderne Gesellschaften sind durch beides geprägt. Wir alle sind in unserem Alltag mit moralischen Urteilen konfrontiert, die sich in vielerlei Hinsicht und grundlegend von unseren eigenen Überzeugungen unterscheiden können - sowohl innerhalb unserer Gesellschaften als auch zwischen den Kulturen.

Kann es gerechtfertigt sein, die eigenen Organe zu verkaufen? Unter welchen Bedingungen ist es erlaubt, an Menschen zu forschen? Dürfen embryonale Stammzellen in der Forschung verwendet oder hergestellt werden? Auf diese und viele andere Fragen gibt es in unterschiedlichen Kulturen verschiedene und oft gegensätzliche Antworten. Darüber hinaus fordern, zum Beispiel, die Praktiken in einigen internationalen Forschungskollaborationen und der internationale Organhandel nicht nur unsere moralischen Intuitionen heraus, sondern bringen uns oftmals auch an argumentative Grenzen. In pluralistischen Gesellschaften und in einer globalisierten Welt scheint sich die Frage nach der Möglichkeit einer kulturübergreifenden Bioethik immer häufiger und dringlicher zu stellen.
Aber ist eine kulturübergreifende Bioethik tatsächlich wünschenswert? Oder ist sie sogar praktisch notwendig? Bestehen faktisch vielleicht schon mehr kulturübergreifende Konsense in bioethischen Fragen, als wir gemeinhin annehmen? Und (wie) lieBe sich eine kulturübergreifende Bioethik normativ begründen? Was wären schließlich geeignete Rahmenbedingungen, um gemeinsame ethische Standards zu formulieren? Dies waren die zentralen Fragen des interdisziplinären Workshops „Zur Möglichkeit einer kulturübergreifenden Bioethik“, der im Frühjahr 2006 auf Initiative von Nikola Biller-Andorno (Zürich), Annette Schulz-Baldes (Zürich) und Peter Schaber (Zürich) am Ethikzentrum der Universität Zürich stattfand. Den etwa 30 deutschsprachigen Wissenschaftlerinnen und Wissenschaftlern aus Philosophie, Sozialwissenschaften, Medizin, Recht und Theologie ging es in dem Workshop nicht nur darum, ob die Wahrheit (bzw. Falschheit) moralischer Urteile oder ihrer Begründungen universal oder aber relativ zum jeweiligen historischen oder kulturellen Kontext ist. Vielmehr wurde das Problem der universalen oder relativen Gültigkeit moralischer Urteile in den Bereich bioethischer Konflik- 
te und damit in die angewandte Ethik getragen. Hierdurch erfuhr eine der klassischen Fragen der Metaethik eine Erweiterung um pragmatische, deskriptive und prozedurale Aspekte. Zentrale Thesen einiger ausgewählter Beiträge sollen im Folgenden vorgestellt werden.

\section{Zur praktischen Notwendigkeit einer kulturübergreifenden Bioethik}

Mit der Wendung zur Praxis stellt sich die Frage nach der Möglichkeit einer kulturübergreifenden Bioethik zunächst auf einer pragmatischen Ebene. In ihrem einführenden Vortrag differenzierte Nikola Biller-Andorno (Zürich) die begrifflichen und argumentativen Grundlagen einer kulturübergreifenden Bioethik und traf ausgehend vom aktuellen Diskurs drei zentrale Unterscheidungen, die sich auch in den verschiedenen Beiträgen des Workshops widerspiegelten. Während eine „kulturübergreifende" Bioethik mit empirisch-sozialwissenschaftlichen und hermeneutischen Methoden die normativen Gehalte aufzufinden sucht, die verschiedenen Kulturen gemein sind, will eine „universale“ Bioethik eine für den Bereich der Medizin und Biologie relevante, allgemeingültige ethische Theorie oder Elemente einer solchen begründen oder anwenden. Eine "globale“ Bioethik geht schließlich von der politischen Notwendigkeit weltweit gültiger Standards im Bereich der Bioethik aus und erarbeitet solche. Am Beispiel des internationalen Organhandels illustrierte Nikola Biller-Andorno, dass bioethische Diskurse parallel auf allen drei Ebenen stattfinden, wobei die Ebenen untereinander vernetzt sind. Robuste globale Standards basieren daher nicht nur auf einem de facto bestehenden kulturübergreifenden Konsens, sondern sind zugleich durch normative Elemente mit universalem Anspruch fundiert. In seinem Kommentar betonte Heiner Roetz (Bochum) bei allen Unterscheidungen nicht $\mathrm{zu}$ vergessen, dass die traditionellen ethischen Theorien empirisch vorfindliche moralische Praktiken und Überzeugungen oftmals nicht hinreichend $\mathrm{zu}$ fassen vermögen und erinnerte daran, dass das „Kulturargument“ insbesondere im politischen Diskurs auch zu einem Werkzeug rein pragmatischer Interessen werden kann.

In der kritischen Auseinandersetzung mit rein pragmatischen Interessen ist in den letzten Jahrzehnten vor allem in der internationalen Politik verstärkt auf die Menschenrechte Bezug genommen worden, und dies auch im Bereich medizinischer Praxis und biowissenschaftlicher Forschung. Roberto Andorno (Zürich) beleuchtete in seinem Beitrag das Verhältnis zwischen internationalem Recht und Bioethik und interpretierte menschenrechtliche Prinzipien - insbesondere die Wahrung der Menschenwürde - als überzeugende und kulturübergreifend akzeptierte Minimalstandards auch für die Bioethik. Die Fülle an entsprechenden internationalen Dokumenten zeige an, dass im Bereich der Biomedizin kulturübergreifender Regelungsbedarf besteht.

Dass ethische Standards faktisch nicht immer gewahrt werden, verdeutlichen die Praktiken in der internationalen biomedizinischen, insbesondere der klinischen Forschung. Internationale Forschungskollaborationen zwischen Industrie- und Entwicklungsländern sind ein Bereich, in dem sich im Umgang mit den Studienteilnehmern in Entwicklungsländern hinsichtlich der informierten Zustimmung, des Behandlungsstandards und der Verpflichtungen nach Abschluss einer Studie schwierige ethische Fragen stellen können - oftmals besonders dann, wenn die Forschung durch pharmazeutische Unternehmen finanziert wird. Eine kulturübergreifend gültige Forschungsethik wäre daher klar wünschenswert, zumal immer häufiger multizentrisch und international geforscht wird. Annette Schulz-Baldes (Zürich) zeigte in ihrem Beitrag auf, wie die Prinzipien der (westlichen) Forschungsethik im Kontext internationaler Forschungskollaborationen in Schwierigkeiten geraten. $\mathrm{Zu}-$ gleich machte sie deutlich, dass die Prinzi- 
pien selbst und ihre Umsetzung nur kaum und im eigentlichen Sinn kulturübergreifend diskutiert werden. Um Studienteilnehmer weltweit vor Ausbeutung oder Missbrauch zu schützen, müssten bestehende Prinzipien und Regeln verstärkt noch hinsichtlich ihrer Begründbarkeit und Anwendbarkeit auf globaler Ebene geprüft werden.

\section{Kulturübergreifende Konsense in der Bioethik?}

Eine im eigentlichen Sinn kulturübergreifende Diskussion in der Bioethik setzt detailliertes Wissen darüber voraus, wie die relevanten normativen Fragen in anderen Kulturen verstanden und diskutiert werden. Vielleicht ließen sich mit empirischsozialwissenschaftlichen und hermeneutischen Methoden sogar implizit kulturübergreifend bestehende, normative Konsense nachweisen? Aber auch ein klareres Verständnis der Differenz würde möglicherweise die internationalen Diskussionen schärfen.

In Afrika zum Beispiel gibt es gar keine (prinzipiengeleitete) Bioethik im westlichen Sinne, so Walter Bruchhausen (Bonn), wenngleich so etwas wie eine „medizinische Moral“ natürlich existiere. Deren Elemente sind aber vorwiegend kultureigen, denn afrikanische Gesellschaften unterscheiden sich in ihren Traditionen und Gesellschaftsstrukturen so stark von westlichen Gesellschaften, dass auch moralische Vorstellungen und Urteile - wie zum Beispiel zum Arzt-Patienten-Verhältnis mit westlichen Normen und ethischen Prinzipien oftmals nur schwer zu vereinbaren sind. Darüber hinaus werde ein Problem einer kulturübergreifend gültigen Bioethik am Beispiel Afrikas besonders deutlich, so Walter Bruchhausen: was in westlichen Journalen als afrikanische „Bioethik“ erscheint, ist keineswegs repräsentativ und oftmals polemisierend verfasst. Für eine kulturübergreifend gültige Bioethik wäre aber ein authentischer kulturübergreifender Diskurs voraussetzend, und es sei eine schwierige Aufgabe, die Beteiligung aller relevanten Parteien praktisch zu gewährleisten.

Auch hinsichtlich des therapeutischen Klonens konnte Phillan Joung (Bochum) weder einen internationalen noch - innerhalb von Südkorea - einen nationalen Konsens feststellen. Die Herstellung embryonaler Stammzellen zu Forschungszwecken ist in Südkorea durch den Fälschungsskandal um den Stammzellforscher Hwang Woo-Suk wieder vermehrt in die öffentliche Diskussion geraten. Dabei ist auch in Südkorea die (kulturübergreifende?) Tendenz zu verzeichnen, dass sich die Debatte inhaltlich von den theoretischen Fragen nach dem moralischen Status des Embryos hin $\mathrm{zu}$ den praktischen Schwierigkeiten der massenhaften und mit gesundheitlichen Risiken verbundenen Rekrutierung von Eizellspenderinnen verschoben hat. Ein auch nur nationaler Konsens in dieser Frage ist aber nicht in Sicht; gleichwohl herrscht in Südkorea aus Gründen der technischen Unzulänglichkeit ein relativer Konsens gegen das reproduktive Klonen.

Dass der Hirntod kein kulturübergreifend gültiges Todeskriterium ist und dies auch nicht sein könne, vertrat Christian Steineck (Bonn). Der Hirntod als Todeskriterium beruhe auf dem Konsens einer medizinischen Expertenkultur und sei in dieser „Subkultur“ durchaus kulturübergreifend anerkannt. Dies begründet aber keineswegs seine kulturübergreifende Gültigkeit im eigentlichen Sinne, denn die Normen einer Expertenkultur können wiederum mit kulturellen oder religiösen Traditionen scharf konfligieren - wie die japanische Debatte um das Hirntodkriterium zeigt. Christian Steinecks Beitrag machte damit zugleich deutlich, dass eine umfassende Definition des Begriffs der „Kultur“ für das Projekt einer kulturübergreifend gültigen Bioethik gleichermaßen notwendig und problematisch ist.

Florian Braune (Göttingen) hinterfragte in seinem Beitrag das Konzept der individuellen informierten Zustimmung im Hinblick auf seine kulturübergreifende Be- 
gründ- und Anwendbarkeit. Gleichwohl global von Bedeutung, wird die Einwilligung für medizinische Eingriffe oder für die Teilnahme an biomedizinischer Forschung in den verschiedenen Gesellschaften sehr unterschiedlich implementiert, sodass es ein kulturübergreifend gültiges Konzept der informierten Zustimmung nicht gibt. Insbesondere das im asiatischen Raum übliche familiäre Zustimmungsmodell kontrastiert stark mit der Praxis der individuellen Zustimmung in westlichen Gesellschaften; in den verschiedenen Zustimmungsmodellen spiegeln sich unterschiedliche Auffassungen von der Autonomie eines Individuums wider. Daher halten manche die familiäre Zustimmung sogar für unvereinbar mit Autonomie, während andere die Angehörigen stärker in die medizinische Entscheidungsfindung einbeziehen wollen. Im deutschen Transplantationsgesetz kommt der Stimme der Angehörigen beispielsweise schon jetzt ein relativ hoher Stellenwert zu, trotz des sonst in Deutschland vorherrschenden individuellen Zustimmungsmodells. Insgesamt ist es in Fragen der informierten Zustimmung aus ethischer Perspektive entscheidend, so Florian Braune, dass der Patient sein „Entscheidungsmodell“ frei wählen kann.

\section{Zur normativen Begründbarkeit einer kulturübergreifenden Bioethik}

Ein Bezug auf kulturübergreifend bestehende normative Konsense scheint also in den konkreten Fragen der Bioethik derzeit offenbar nur schwer möglich zu sein. Machen Pluralismus und Globalisierung eine kulturübergreifend gültige Bioethik dennoch praktisch notwendig, stellt sich die Frage, ob und wie sich eine solche normativ begründen ließe. In mehreren Beiträgen des Workshops wurden verschiedene philosophische Ansätze und normative Theorien daraufhin untersucht, ob und unter welchen Bedingungen sie die normative Fundierung einer kulturübergreifend gültigen Bioethik darstellen könnten.
Jedem normativen Begründungsversuch in der Bioethik muss aber, so Anton Leist (Zürich), im Hinblick auf das jeweils zugrunde gelegte Personenmodell eine Selbstaufklärung der sich darin widerspiegelnden moralischen Intuitionen vorangehen. In Abhängigkeit davon, ob - im europäischen Kontext - von einem aristotelisch-stoischen, christlichen oder aufklärerischen Modell der Person ausgegangen wird, kämen wir in bioethischen Fragen nämlich zu ganz unterschiedlichen Antworten. Dies zeigt sich vor allem an unseren Auffassungen zum Umgang mit Lebensbeginn und Lebensende, aber auch hinsichtlich der Beziehung zwischen Patient und Gesellschaft und der Gestaltung des Körpers. Für die europäische Bioethik der letzten Jahre diagnostizierte Anton Leist eine praktische Wende hin zu einer Art aristotelisch-stoischem, an der Klugheit orientiertem Personenmodell; diese Praxisorientierung könnte als die Grundlage dafür angesehen werden, Patientinnen und Patienten als die eigentlichen Experten der Medizinethik zu verstehen.

Völlig ohne vorausgehende Verständigung in substantiellen Fragen, sondern rein prozedural versteht dem gegenüber Herlinde Pauer-Studer (Wien) eine kulturübergreifend gültige Bioethik. Denn in vielen bioethischen Kontroversen ist die Einigung über Verfahren der Entscheidung von substantiellen Fragen alles, was sich an Konsens erreichen lässt. Erst über vorher klar definierte Verfahren ist dann - in Anknüpfung an John Rawls - ein überlappender Konsens auch in inhaltlichen Fragen möglich. Norbert Anwander (Zürich) hinterfragte in seinem Kommentar kritisch, ob durch einen überlappenden Konsens nicht allenfalls politische Kompromisse erreicht werden und wies darauf hin, dass gute politische Lösungen nicht schon zu richtigen moralischen Urteilen führen.

Mit der Diskursethik stellte Niels Gottschalk-Mazouz (Stuttgart) einen anderen prozeduralen, eigentlich aber rationalistischen Begründungsansatz für eine kulturübergreifend gültige Bioethik vor. Der 
Diskursethik zufolge ist genau diejenige Handlungsweise moralisch richtig, der alle Teilnehmer eines zwanglos geführten argumentativen (rationalen) Diskurses zustimmen können. Aber ist dieses diskursethische Wahrheitskriterium - und damit der Vernunftbegriff, auf dem die Diskursethik basiert - auch kulturübergreifend gültig? Niels Gottschalk-Mazouz schien diese Frage grundsätzlich zu bejahen, sah aber eine Reihe von Schwierigkeiten in der praktischen Umsetzung eines weltweiten bioethischen Diskurses: möglicherweise gibt es zwischen Kulturen zu wenig inhaltliche Gemeinsamkeiten, um überhaupt ins Gespräch treten zu können, und Menschen, die anti- oder adiskursiven Kulturen zugehören oder in totalitären Staaten leben, können an einem solchen Diskurs erst gar nicht teilnehmen.

Nicht auf die Praxis eines argumentativen Diskurses, sondern auf die deliberative Prüfung unserer wohlüberlegten moralischen Überzeugungen setzt in der Bioethik der einflussreiche prinzipienethische Ansatz von Beauchamp und Childress. Der Prinzipienethik zufolge entspringen moralische Überzeugungen der common morali$t y$, die als Kern universal gültiger Normen, Rechte und Tugenden von allen moralisch ernsthaften Menschen geteilt und damit kulturübergreifend anerkannt wird. An dieser Stelle würde sich natürlich ein Ansatzpunkt für eine kulturübergreifend gültige Bioethik anbieten. Oliver Rauprich (Nürnberg-Erlangen) vertrat in seinem Beitrag allerdings, dass in der Prinzipienethik die Konzeption einer common morality zugunsten einer kohärenten Rechtfertigung moralischer Urteile im Überlegungsgleichgewicht aufgegeben werden muss. Zwar sei die empirische These der Existenz einer gemeinsamen Moral durchaus plausibel, wie auch der breite internationale Konsens hinsichtlich der Menschenrechte anzeige, aber diese könne nicht die Gültigkeit moralischer Urteile begründen. Nur wenn sie sich an das Überlegungsgleichgewicht und damit an ihre kohärenztheoretischen Wurzeln hält, kann die Prinzipienethik eine kulturübergreifend gültige Bioethik theoretisch fundieren.

Dezidiert an die empirisch vorfindliche Moral und deren Vielfalt knüpfte dem gegenüber Margit Sutrop (Tartu) an. Aber der Pluralismus moralischer Werte impliziert keineswegs einen ethischen Relativismus. Wenn sich bestimmte moralische Regeln und Normen zwischen den Kulturen und auch innerhalb einer Kultur über die Zeit verändern, dann ist dies auf die unterschiedliche Interpretation universaler Werte - wie Aufrichtigkeit, Freiheit, Menschenwürde, Gerechtigkeit und Wohltun und nicht auf die Anerkennung unterschiedlicher Werte zurückzuführen. Auch in der Diskussion beispielsweise um die Stammzellforschung ist unumstritten, dass menschliches Leben einen Wert darstellt. Aber unterschiedliche Interpretationen der Menschenwürde resultieren in unterschiedlichen Wertkonflikten, die unterschiedlich aufgelöst werden können und so zu einem Wertepluralismus führen. Die Aufgabe einer kulturübergreifend gültigen Bioethik ist es folglich, die Vorzüge oder Richtigkeit einer bestimmten Interpretation der universalen Werte argumentativ auszuweisen.

$\mathrm{Zu}$ fragen, ob und wie verschiedene ethische Theorien eine kulturübergreifend gültige Bioethik begründen könnten, macht nur Sinn, wenn Moral überhaupt universal begründbar ist. Peter Schaber (Zürich) widmete sich in seinem Beitrag der Gegenthese zu jeder wie auch immer fundierten kulturübergreifend gültigen Bioethik: der (metaethischen) These des ethischen Relativismus, dass moralische Urteile nur relativ zu einer Gruppe, Gesellschaft oder Kultur wahr bzw. falsch sind. Der ethische Relativismus ist aber in keiner seiner Formulierungen eine kohärente Doktrin, so Peter Schaber. Dies gilt auch für den häufig vertretenen partiellen Relativismus, nach dem es neben universalen auch kulturrelative Normen gibt. Denn der partielle Relativismus beruht auf der falschen Voraussetzung, dass moralische 
Normen aus Gründen der sozialen Anerkennung verbindlich sind. Soziale Anerkennung kann hingegen immer nur die Verbindlichkeit sozialer, nicht aber die Verbindlichkeit moralischer Normen begründen. Aus diesem Grund ist der ethische Relativismus zurückzuweisen und das Projekt einer universalen Bioethik grundsätzlich möglich.

\section{Rahmenbedingungen \\ für die Formulierung gemeinsamer ethischer Standards unter den Bedingungen des Pluralismus}

Halten wir als vorläufiges Ergebnis des Workshops fest: eine kulturübergreifend gültige Bioethik wäre in vielerlei Hinsicht wünschenswert - und in manchen Bereichen der Biomedizin erscheint sie sogar praktisch notwendig -, aber ein Rekurs auf (implizit) kulturübergreifend bestehende Konsense hinsichtlich konkreter bioethischer Fragen ist derzeit nicht möglich, und bezüglich der normativen Begründung einer kulturübergreifenden Bioethik gibt es wie $\mathrm{zu}$ erwarten unterschiedliche Positionen. Wie können unter diesen Bedingungen dennoch gemeinsame Standards formuliert werden?

Im Lichte ihrer Erfahrungen in konfessionellen, nationalen und internationalen bioethischen Kommissionen und Gremien reflektierten Andrea Dörries (Hannover), Claudia Wiesemann (Göttingen) und Andreas Reis (Genf) diese Fragestellung in der letzten Sitzung des Workshops. Dabei wurde deutlich, dass sich in der Praxis kulturübergreifend gültige inhaltliche Konsense $\mathrm{zu}$ bioethischen Fragen - wie beispielsweise hinsichtlich Stammzellforschung oder Verteilungsgerechtigkeit im Gesundheitswesen - am ehesten auf der Basis eines „prozeduralen Konsenses“ erarbeiten lassen. Prozedurale Rahmenbe- dingungen sind zum Beispiel allgemeine Regeln zur Konsensfindung, aber auch zum expliziten Umgang mit Dissensen (Andrea Dörries) oder das auf Norman Daniels zurückgehende Konzept eines „fairen Prozesses“, das sich an der Weltgesundheitsorganisation bewährt hat (Andreas Reis). Dabei stellt sich natürlich immer die Frage, ob moralische Konsense nicht $\mathrm{zu}$ bloß politischen werden, und ob bei der Konsensfindung kontroverse Positionen und verschiedene politische Kulturen hinreichend Berücksichtigung erfahren. Von der Aushandlung konkreter und kulturübergreifend gültiger normativer Konsense im Bereich der Biomedizin müsste daher mehr noch an der ethischen und politischen Spezifizierung bioethischer Kommissionen und Gremien selbst gearbeitet werden (Claudia Wiesemann).

Somit zeigte sich auf dem Workshop in Zürich nicht nur, dass Pluralismus und Globalisierung eine kulturübergreifend gültige Bioethik in einigen Bereichen praktisch notwendig machen. Zugleich wurde auch deutlich, wie breit das Spektrum der theoretischen und praktischen Schwierigkeiten ist, die auf dem Weg dorthin erwartet werden können. Dass der hierfür notwendige kulturübergreifende Diskurs zumindest zwischen den Fächern möglich und darüber hinaus auch bereichernd und konstruktiv ist, zeigten die drei intensiven Tage in Zürich. Die Beiträge dieser gelungenen Veranstaltung werden bis 2007 in einem Sammelband herausgegeben.

\section{Anschrift}

\section{Dr. med. Annette Schulz-Baldes}

Lehrstuhl für Biomedizinische Ethik

Ethik-Zentrum der Universität Zürich

Zollikerstr. 115

8008 Zürich, Schweiz 